\title{
On the Possibility of Emotional Robots
}

\author{
Sobre a Possibilidade de Robôs Emocionais
}

GODWIN DARMANIN ${ }^{a}$

\begin{abstract}
In this article, I examine whether the possibility exists that in the foreseeable future, robot technology will permit the development of emotional robots. As the title suggests, the content is of a technological as well as of a philosophical nature. As a matter of fact, my aim in writing this paper was that of bridging two distinctive fields in a world where humanity has become accustomed to technological innovations while overlooking any consequential complications arising from such inventions. To this end, I review and commentate on what Anders Sandberg, Paul Thagard, Nikhil Churamani and other thinkers have contributed on the subject matter. The literature review indicates that in the short to the medium term, scientists will only design and engineers will only build robots that will be able to only learn, be trained, or under the most optimistic conditions only simulate human emotions. However, in the long term the possibility exists for technology to advance to such a state so as to permit an entire human brain to be emulated in a robot via a concept named 'mind uploading'. If one day that becomes a reality, that will be the point where humanity will possibly come closest to creating robots with emotions.
\end{abstract}

Keywords: Affective computing. Artificial intelligence. Emotions. Emotional intelligence. Robot technology.

\section{Resumo}

Neste artigo, examino se existe a possibilidade de que, no futuro previsível, a tecnologia robótica permita o desenvolvimento de robôs emocionais. Como o título sugere, o conteúdo é de natureza tanto tecnológica quanto filosófica. De fato, meu objetivo ao escrever este artigo foi o de unir dois campos distintos em um mundo em que a humanidade se acostumou às inovações tecnológicas enquanto ignorava as complicações resultantes de tais invenções. Para esse fim, reviso e comento as contribuições de Anders Sandberg, Paul Thagard, Nikhil Churamani e outros pensadores sobre $o$ assunto. A revisão da literatura indica que, a curto $e$ médio prazo, os cientistas somente projetarão e os engenheiros construirão robôs que só

\footnotetext{
a Sofia University, Sofia, Bulgaria. Doutor, e-mail: godwin.darmanin@gmail.com
} 
poderão aprender, ser treinados ou, nas condições mais otimistas, simular apenas as emoções humanas. No entanto, a longo prazo, existe a possibilidade de a tecnologia avançar para tal estado, de modo a permitir que um cérebro humano inteiro seja emulado em um robô por meio de um conceito chamado 'carregamento mental'. Se um dia isso se tornar realidade, esse será o ponto em que a humanidade possivelmente se aproximará da criação de robôs com emoções.

Palavras-chave: Computação afetiva. Inteligência artificial. Emoções. Inteligência emocional. Tecnologia de robôs.

"Humans aren't machines. We feel, we love, we cry, we despair, and we rejoice. Anyone who's ever tried to convince me not to feel is someone I shouldn't have trusted. The only reason you should shut off your emotions and emulate a robot is if you're doing horrible things".

(Forlorn Passions by Bruce Crown).

\section{Introduction}

One of the main characters of the neo-noir science fiction film Blade Runner was Rachael, a prototype female replicant. Since engineers had implanted in her, recollections belonging to the niece of her human creator, she initially believed that she was human. However, she eventually learns that she is not truly human and yet, she falls in love with the other character, Blade Runner Rick Deckard. Rachael was such an advanced humanoid robot, resembling and acting exactly like humans, that she even conceived a child with Deckard. From Blade Runner 2049, the highly anticipated sequel of the original film, we also learn that she had died as a result of the birth of their child Ana.

Although the possibility of robotic procreation is certainly too farfetched at the present, one cannot entirely exclude the possibility that one day such an occurrence becomes a reality. Nonetheless, irrespective of how much intriguing such a possibility could prove to be, my aim in writing this article is not that of exploring such grandiose futuristic technologies. Rather, in this article I ponder on robots from the perspective of the Philosophy of Emotions. As regards the latter, one can observe that in recent decades, emotions have emerged as an area of prime concern in philosophy and while the latter appears to be a rather new development, attention to emotions has a far longer, and more 
lasting history, reaching back to the earliest beginnings of philosophical inquiry itself. For instance, the great philosophers of antiquity such as Plato and Aristotle, as well as $17^{\text {th }}$ century philosophers such as Spinoza and Descartes were very much intrigued by emotions.

The emotional relationship which had developed between Rachael and Deckard fashioned the emotive crux of the original Blade Runner. Accordingly, given the fact that emotions have been a common preoccupation of major philosophical traditions, regardless of time and space, I examine the possibility that one day robots will also have emotions.

\section{What are Emotions?}

Before embarking on a discussion on the possibility of robots having emotions, one ought to define to begin with, what emotions are and here we encounter the first problem. In this regard, Klaus R. Scherer acknowledges the fact that defining 'emotion' is a notorious problem and adds that without a consensual conceptualisation and operationalisation of exactly what phenomenon is to be studied, progress in theory and research is difficult to achieve and that futile debates are likely to flourish. Scherer further adds that William James had already tried to give a convincing answer in 1884, only to start an enduring debate. In fact, Scherer observes that the number of scientific definitions proposed has grown to the point where counting has become quite hopeless, with more than one hundred already counted in a review published in 1981 by Kleinginna and Kleinginna (SCHERER, 2005).

More recently, Andrea Scarantino and Ronald de Sousa observed that 'Emotion' is a term that came into use in the English language in the seventeenth and eighteenth centuries as a translation of the French term émotion. However, they state that until the midnineteenth century the term itself did not refer to a category of mental states that might be systematically studied. Nonetheless, they also highlight the fact that many of the things that nowadays we call emotions have since Ancient Greece been the object of theoretical analysis, albeit under different terms such as sentiment, passion, affection, disturbance, affect, movement, perturbation, upheaval and appetite (SCARANTINO \& SOUSA, 2018). 
All of these dissimilar terms can lead us to perceive emotions as eliciting different meanings to different people or in different circumstances. However, as John Deigh observed a decade earlier, two principal themes have characterised the study of emotions in modern philosophy and psychology. One of these themes identifies emotions with feelings, whereas the other treats emotions as states of mind that are directed in the direction of some object. Within the context of the first theme, emotions are conceptualised as feeling-centered, whereas in the second theme emotions are conceptualised as thought-centered (DEIGH, 2009).

We can differentiate between the two concepts with an example which takes its inspiration from the subject matter of this paper. In this regard, let us take into consideration 'fear' and examine it in accordance with the first theme which was originally conceptualised by William James (JAMES, 2017). The police department of a technologically advanced society deploys a robotic police officer to chase criminals in situations which are considered to be too dangerous for human police officers to handle. One day, while on duty, the robotic police perceives an attack by a human criminal through the use of a grenade launcher. The robot utilises AI (Artificial Intelligence) to analyse the situation and takes into account external factors such as the physical environment, the type and the size of the weapon and the maximum force that the human criminal can exert. Let us assume that following the analysis, the robot determines that it would be futile to confront the human criminal and that the best course of action is that of fleeing the scene. According to this first theme it is the feeling of such bodily movement that is the 'fear'. Thus according to this theme, emotions are epiphenomenal. This implies that as mental events, they are entirely dependent (for their presence) on one or more matching physical and biochemical events which would need to happen within the robotic body, and that the same mental events would have no causal effect on physical events. Now, as far as we know, there is at present not even one single robotic body which can internally experience biochemical events. Nonetheless, one cannot totally exclude the possibility that such a machine could be eventually built.

On the other hand, in order to elucidate the second theme, let us imagine the same situation, that is the same robotic police that is being confronted by the same human criminal carrying the same heavy weapon. Can the robot, perceive the criminal as an object which induces fear, with the intentionality of the emotion consisting in it directing the 
robot towards the criminal? Answering such a question poses a problem since even though one would certainly expect the robot to carry out its duty and perceive the criminal as someone who induces fear in law abiding citizens, can the robot itself perceive that fear in the same way as a carbon based human being would perceive it?

\section{The need for Robots to have Emotions}

As amply demonstrated through the previous example, it is highly desirable that on a practical level, robots experience certain emotions. If for instance, the robotic police depicted in our example cannot feel any fear when confronted by the criminal holding the grenade launcher, it would need to rely on very accurate visual and environmental sensors together with advanced AI to determine that an escape is the best course of action. Emotions would also come in handy when the same robotic police would need to attempt a heroic approach while confronting certain life-threatening situations. After all, it is highly appreciated and usually even expected by the general public to have police officers perform heroic acts.

In this regard, Paul Thagard observes how modern technology is developing in such a way as to permit autonomous robots to emulate the processing of information and make decisions in ways that are more and more comparable to those of human brains (THAGARD, 2012). Thagard draws from the realms of computer science and computer hardware and states that FPGA (Field Programmable Gate Arrays) technology can be utilised to simulate the computational, representational and dynamical aspects of neural activity. Accordingly, Thagard imagines the possibility that one day robots will have in them implemented a sophisticated hormonal adjustments system based on emotions. He asserts that this could be achieved by letting the robot's hardware to be reprogrammed by a controlling unit on an ad-hoc basis. Thagard affirms that such a system would operate on the basis of past emotional states, the associated actions and their outcomes, as well as the environment.

The type of system envisaged by Thagard is not an implausible one. As a matter of fact, it does not even require some kind of ground-breaking technology which does not presently exist. Rather, one of the chief obstacles for the implementation of a state of the 
art version of such a system is the presumably large amount of computing storage required to store all of the emotional states, the associated actions and the outcomes required to allow the robot to take the best course of action. Moreover, the robot's artificial memory would need to be also crammed with environmental imagery of all types of terrain where the robot will operate, something which would also require lots of storage. Nonetheless, such a system would only imitate the neural structure and functioning of a human brain and would certainly not operate efficiently if the robot encounters an ill-defined terrain or a vague situation.

\section{Emotional Expressions in Robots}

It is conventionally accepted that facial expressions are movements which convey the emotional state of a human being (and of some animals) to observers. Hence, most of the times (although not necessarily) when humans interact with each other, they also express their emotions through facial expressions. Thus, a fellow human being who belongs to the same family or wider society would immediately recognise if the other person is for instance either afraid or surprised. Accordingly, one would certainly expect a robot to convey facial expressions if that robot is to express emotions in an authentic way.

Qin Huang et al. (2018) affirm that to give robots the ability of emotional expression is a major research topic in robotics. They then proceed with mentioning a number of real world examples of robots capable of facial expressions, such as Kismet (a humanoid robot capable of expressing six types of facial expressions), the robot SAYA (who generates facial expression based on pneumatic artificial muscles) and the H\&F robot-I who has the capacity of showing eight basic emotions. As regards the latter, Hao Guan et al. (2011) tell us that this robot succeeded to realise six (not eight) basic facial expressions and that these expressions are ones which portray neutrality, happiness, anger, disgust, sadness and surprise. However, they also add that these six basic expressions are not comparable with those expressed by real people since they are not natural and vivid enough.

Another issue to consider is the fact that for robot facial expressions to be truly natural, they should not take place in isolation. This implies that a robot which can truly display facial expressions should do so within the context of interactions with human 
beings or with other robots. In this regard, Huang et al. (2018) assert that a humanoid robot with the name NAO has an emotional and behavioural learning mode which can analyse human emotion and interact with people bilaterally through the observation of their expression and poses.

The above implies that a technologically advanced and sophisticated robot can be induced to observe humans and learn from them. At this point, a pertinent question arises as to whether humans themselves learn facial expressions from others, perhaps during their infancy. In this regard, David Natsumoto and Bob Willingham carried out research on the spontaneous facial expressions of emotion of congenitally and noncongenitally blind individuals. Their findings offer convincing evidence that naturally fashioned facial emotional expressions of visionless persons are the same as those of sighted people, when expressed in the same emotionally evocative conditions. Moreover they also add that they function in the same ways. They also strongly suggest that the universality in emotional expressions observed in several studies that involve humans, originates from an evolved, possibly genetic source and that all humans, irrespective of gender or culture, are born with this capability. Indeed, they arrived at this conclusion because blind people, particularly those born blind, could not conceivably have learned to produce those precise facial arrangements from modelling the expressions of others in culture-constant learning (MATSUMOTO and WILLINGHAM, 2009).

Thus, from the above we can infer that in relation to the subject matter of this paper, technologically advanced robots can learn, either from humans or possibly from other robots with similar capabilities, how to reproduce emotional expressions. Nonetheless, such an inference does not preclude the possibility that scientists program the facial expressions into the robot's artificial memory, that engineers implement a set of dependant artificial muscles and that through AI the robots will be able to interact 'naturally' with human beings without the need to learn from them which facial expression to execute within a particular context. However, in this regard, one could still possibly argue that if such a mechanism is put in place, the robot would have just 'learned' the facial expressions from the scientists and that consequently this is not comparable with the innate capabilities of human beings. 


\section{The Affective Robot}

"Do you know what it feels like to be in love with someone that is about to marry someone else?". These are the words uttered by Andrew Martin, in the 1999 science-fiction comedy-drama film 'Bicentennial Man', which in turn is based on the novel 'The Positronic Man' by Isaac Asimov and Robert Silverberg (ASIMOV and SILVERBERG, 1993). Andrew Martin was a human-looking robot. However, he could also be defined as an affective robot, a type of robot fashioned on technology that derives from Affective Computing. The latter is a branch of computing that is concerned with the theory and construction of machines which can detect, respond to, and simulate human emotional states (ENGLISH OXFORD LIVING DICTIONARIES, 1990).

In this regard, Nikhil Churamani et al. (2018) assert that being able to identify emotions in human users is considered a highly desirable attribute in Human-Robot Interaction (HRI) situations. However, they also add that most present-day approaches hardly attempt to apply identifiable emotional features in an active way, so as to modulate robot decision-making and dialogue for the profit of the human user. In their position paper, they propose a method of incorporating recognised emotions into a Reinforcement Learning (RL) based dialogue management module. The latter is an approach to machine learning that is inspired by behaviourist psychology and which allows a machine to learn in a way that is similar to how a child learns to accomplish a new task. In this way the affective robot can adapt its dialogue as it learns different emotional expressions in order to empathise with elderly people while these are playing a puzzle game. What they propose is certainly admirable but at the same time not so straightforward to implement. In practice they suggest an interaction scenario which involves the subjects (the elderly) playing several rounds of the 2048 Puzzle Game with Nao the robot.

They suggest that $\mathrm{NaO}$ is to be pre-trained (through the use of Reinforcement Learning techniques) with procedures that aim to solve the game while at the same time providing suggestions to the elderly on the best move required to succeed in the game. Such suggestions shall be determined according to the user's emotional state. Thus, if for instance the elderly are feeling positive and performing well in the game, the robot will concentrate on further improving their performance by enthusiastically providing feedback 
(spoken and gestural) about their moves in the game. On the other hand, if the elderly are feeling negative or irritated, Nao will direct its energies on them so that they make an effort to improve their emotional state. Essentially, the robot proposed by Churamani et al shall adapt its conduct to encourage the users to complete the tasks by offering them assistance and by helping them cope with the frustration that brain teaser games usually provoke. Since the proposed technique that is to be used allows the robot to learn in a similar way to how a child learns, one can argue that in such a scenario the robot is exhibiting behaviour which is on par to human behaviour. Hence, in contrast to expressing facial expression, affective behaviour in robots seems more promising. Nevertheless, having a robot behaving in suitable manner because it has learnt expected behaviour does not imply that the robot is at the same time showing authentic empathy.

\section{Artificial Emotional Intelligence and Self-Awareness}

The previous discussion paves the way to the notion of Emotional Intelligence, which Jeff Feldman and Karl Mulle define as "Using your emotions intelligently to gain the performance you wish to see within yourself and to achieve interpersonal effectiveness with others" (FELDMAN and MULLE, 2007). The authors acknowledge the fact that theirs is a concise definition. However, they also expound on their definition by stating that using one's emotions implies both awareness of and the ability to manage those emotions. They further add that using one's emotions intelligently means that one can consciously reflect on his emotions and then choose appropriate responses. Thus, at this point the question which arises is whether robots will one day become aware of their emotions, whether they will be able to manage them, whether they will be able to consciously reflect on their emotions and whether (as a consequence) be able to choose apposite responses.

Lavanya Rathnam believes that self-aware robots could one day become a reality, when one considers the many experiments that are being done in this area. In this regard she mentions the mirror test, a classic test for self-awareness, which assesses the ability to recognise one's own reflection in a mirror (RATHNAM, 2018). In reference to this, Justin Wildrick Hart affirms that the mirror-test has become the de facto standard by which an animal is judged to be self-aware and that it consequently provides both a recognisable 
breakthrough in the development of self-aware artificially-intelligent systems and a stimulating target for research into their development (WILDRICK HART, 2014). Both Rathnam and Wildrick Hart refer to the robot named Nico, which was developed by scientists at Yale University and which at an early stage was already capable of recognising its arm. Rathman observes that this successful experiment paves the way for producing advanced robots that can realise when some part of their mechanical body is damaged or is not working correctly, so that they can possibly even repair it themselves.

Rathman also mentions an experiment conducted by Professor Selmer Bringsjord and his team at Rensselaer Polytechnic Institute (BRINGSJORD et al., 2015). In this experiment, the researchers gave an updated version of the wise-men puzzle to three Aldebaran Nao humanoid robots, and one of them passed it. Nevertheless, the same Rathman admits that all of this does not imply that robots have the same level of selfawareness as humans, but only that such experiments show that it is possible to train robots to be self-conscious in specific situations.

\section{Man becomes Robot}

The children of the late 70s and 80s grew up watching Japanese anime (animated media) series such as Cyborg 009 (orginal name: Saibōgu Zero-Zero-Nain) and Galaxy Express 999 (original name: Ginga Tetsudō Surī Nain). The main characters in the former series were originally human beings which were converted into cyborgs (beings with both organic and biomechatronic body parts) possessing superhuman powers. On the other hand, the latter series is set in a space-travel, high-tech future in which humans have mastered the technical know-how which allows them to transfer their minds and emotions (with precise fidelity) into robotic bodies, thus in practice allowing them to attain immortality.

Around four decades have passed since then, and such a feat remains obviously confined within the realm of science-fiction. In fact, very recently the same theme has been used in TV series and cinematic films such as in the Altered Carbon series and in the film Transcendence, starring Johnny Depp. This shows that the motif (referenced by various terms such as mind uploading, brain uploading, mind copying or mind transfer) remains 
intriguing for the imagination of contemporary sci-fi aficionados. But will such a surreal fantasy ever become a reality?

Incidentally, the whole idea behind mind uploading poses two types of problems: technical and philosophical. From a technical point of view, Anders Sandberg (Research Fellow at the Future of Humanity Institute at Oxford University) states that presently a practical implementation is impossible since we need not just great nanotechnology but also a fine understanding of how to interface brains to electronics on a truly massive scale (ELDERKIN, 2018). And from a philosophical perspective, Sandberg asks whether this is a transfer of personal identity, some kind of cloning/copying (in order to make a new person with the same or different identity) or something else entirely. In this regard, he states that he is in agreement with the philosopher Derek Parfit who examined similar cases in his book 'Reasons and Persons' (PARFIT, 1984) and who had observed that there is no true fact of the matter about who is the 'real' continuation of the original person, but that what matters is at most psychological connectedness.

In relation to the subject matter of this paper, I think that if one day such advanced technology becomes a reality, the resultant robot would certainly not be human but that nevertheless it will possess human-like characteristics such as rationality and even emotions. Indeed, if it will be the case that the human brain (the source) is entirely and accurately emulated in a robot, that would most probably be the closest that we will ever achieve as regards robots having emotions.

\section{Conclusion}

Throughout this article, I have provided an exposé of what various thinkers have so far contributed in relation to the likelihood that robots might one day experience emotions. As regards the present situation, it was established that current technology only allows for experimentation. Moreover, the fact that robots are machines poses certain practical limitations since for instance at present not even one single robotic body can internally experience biochemical impulses. Nevertheless, technology is progressing in such a way as to allow autonomous robots to emulate the processing of information and make decisions in ways that are relatively analogous to those of human brains. One plausible method to 
achieve this is through the simulation of the computational, representational and dynamical aspects of neural activity. Another proposed technique is the implementation of a hormonal adjustment system based on emotions. This can be achieved via reprogrammable hardware that controls a robot according to the situation. Such a system would function by taking as inputs: past emotional states, the associated actions and their outcomes, as well as the environment.

The ability for robots to have facial expressions was also discussed and in this regard, it was observed that although robotic facial expression technology already exists, the resultant facial expressions are not yet natural and vivid enough as those expressed by human beings. In this regard, it was established that technologically advanced robots will eventually be able to learn from others how to reproduce emotional expressions. Indeed, Human-Robot interaction which is both natural and authentic is also an essential prerequisite within the context of robotic emotions. It was proposed that this can be achieved through Reinforcement Learning (RL), an approach to machine learning that is inspired by behaviourist psychology.

As already stated, various pertinent experiments are being conducted. Some of these are aimed at investigating the possibility of robots having the same level of self-awareness as human beings. However, results achieved so far show that presently we can only conceive of robots which can be trained to be self-conscious in explicit situations. Nonetheless, the idea also exists for one day to have an entire human brain emulated in a robot via a concept termed 'mind uploading'. In this regard, it has been determined that although present-day technology does not currently permit for a human mind to be copied to a robot, if one day the underlying technology is perfectly mastered, that would most possibly be the best that humanity will ever attain as regards creating robots with emotions. If that ever happens, that would certainly also mean opening a Pandora's box that will instigate an emergence of various philosophical problems. Moreover, such a concept might become possible only in the long-term. Thus, in the short to the medium term, we can only envisage robots that will be able to only learn, be trained, or under the most favourable conditions only simulate human consciousness and emotions. This is the case, since consciousness also implies the provision of a response of the Self to the presence of the Other while transcending the world and its objects. Accordingly, human consciousness is not found in the brain of a human, ready to be transferred to a machine. Indeed, human 
consciousness primarily invokes a relation with or an attitude toward the Other. Thus in this regard, our brain is merely a material mediator that supports the invisible connection of the Self with all that it transcends. Therefore, due to its invisible nature (it is neither objectively present nor made up of cells in the brain), I have serious doubts as regards the possibility that consciousness itself could ever be uploaded to a robot. Hence, in the final analysis, that is why the best that we can ever hope for is the production of state-of-theart simulacra.

Consequently, robots incorporating 'mind uploading' technology, would certainly not be exact replicas of human beings but they will nevertheless possess human-like characteristics. Nevertheless, why should humans struggle to create humanoid robots which are virtually identical to humans in every aspect? In spite of everything, ingenuity implies that we create something new that will be capable of helping mankind to prosper, and not that at all costs we create replicants that resemble us in every single aspect. At the same time, we ought not to forget that human creativity must be also kept in check by human responsibility.

\section{References}

ASIMOV, I.; SILVERBERG, R. The Positronic Man. New York: Pan Books, 1993.

BRINGSJORD, S. et al. Real Robots that Pass Human Tests of Self-Consciousness. In: 24th IEEE International Symposium on Robot and Human Interactive Communication (RO-MAN). Anais... Kobe, Japan, 2015.

DEIGH, J. Concepts of Emotion in Modern Philosophy and Psychology. In: Peter Goldie (ed.), The Oxford Handbook of Philosophy of Emotion. Oxford University Press, 2009.

CHURAMANI, N. et al. An Affective Robot Companion for Assisting the Elderly in a Cognitive Game Scenario. In: Proceedings of the Workshop on Intelligent Assistive Computing (WLAC), IEEE World Congress on Computational Intelligence (WCCI) 2018. Available at: $<$ https://www.researchgate.net/publication/326186953_An_Affective_Robot_Companion_for _Assisting_the_Elderly_in_a_Cognitive_Game_Scenario>. Accessed 8 November 2018.

ELDERKIN, B. Will We Ever Be Able to Upload a Mind to a New Body? 2018. [Online] Available at: https://gizmodo.com/will-we-ever-be-able-to-upload-a-mind-to-a-new-body-1822622161. Accessed 8 November 2018. 
ENGLISH OXFORD LIVING DICTIONARIES. Affective Computing. 1990. [Online] Available at: https://en.oxforddictionaries.com/definition/us/affective_computing. [Accessed 2 November 2018].

FELDMAN, J.; KARL, M. J. Put Emotional Intelligence to Work, pp. 10. Alexandria, Virginia, USA: ASTD Press, 2007.

GUAN, H. et al. Mechanism Design of a Facial Robot. In: Chen Ran (ed.), Procedia Engineering, v. 15, p. 416-420, 2011.

HUANG, Q. et al. Robot Vision Navigation Based on Improved ORB Algorithm. In: Fatos Xhafa et al (ed.), Advances in Intelligent Systems and Interactive Applications: Proceedings of the $2^{\text {nd }}$ International Conference on Intelligent and Interactive Systems and Applications (IISA 2017), pp. 127-128. New York, USA: Springer, 2018.

JAMES, W. The Principles of Psychology, Volumes 1-2. First published in 1890. Scotts Valley, California, USA: CreateSpace Independent Publishing Platform, 2017.

MATSUMOTO, D.; Willingham, Bob, 2009. Spontaneous Facial Expressions of Emotion of Congenitally and Noncongenitally Blind Individuals. In: Journal of Personality and Social Psychology, vol 96, n. 1, pp. 1-10.

PARFIT, D. Reasons and Persons. Oxford, New York: Clarendon Press, 1984.

RATHNAM, L. Self-Aware Robots: Science-Fiction Or Reality? [Online]. 2018. Available at: $<$ http://techgenix.com/self-aware-robots>. Accessed in: 7 nov. 2018.

SCARANTINO, A.; SOUSA, R. de. Emotion. In: Edward N. Zalta (ed.), The Stanford Encyclopedia of Philosophy (Winter 2018 Edition). [Online]. 2018. Available at: https://plato.stanford.edu/archives/win2018/entries/emotion. [Accessed 29 October 2018].

SCHERER, K. R. What are emotions? And how can they be measured? In: Social Science Information, vol 44, n. 4, pp. 695-729, 2005.

THAGARD, P. The Role of Emotions in Human Cognition and Robotics. [Online]. 2012. Available at: https://www.academia.edu/2813647/The_Role_of_Emotions_in_Human_Cognition_and_Rob otics. [Accessed 31 October 2018].

WILDRICK HART, J. Robot Self-Modeling. Candidacy for the Degree of Doctor of Philosophy. New Haven, Connecticut, USA: Yale University, 2014. 